\title{
Carbohydrate Metabolism Changes in Prunus persica Gummosis Infected with Lasiodiplodia theobromae
}

\author{
Z. Li, L. Gao, Y. T. Wang, W. Zhu, J. L. Ye, and G. H. Li
}

First, second, third, fourth, and sixth authors: Key Laboratory of Horticultural Plant Biology, and fifth author: National Key Laboratory of Crop Genetic Improvement, Huazhong Agricultural University, Ministry of Education, Wuhan, 430070, China. Accepted for publication 13 November 2013.

\section{ABSTRACT}

Li, Z., Gao, L., Wang, Y. T., Zhu, W., Ye, J. L., and Li, G. H. 2014. Carbohydrate metabolism changes in Prunus persica gummosis infected with Lasiodiplodia theobromae. Phytopathology 104:445-452.

Peach gummosis represents a significant global disease of stone fruit trees and a major disease in the south peach production area of the Yangtze River of China. In this study, the carbohydrate composition of peach shoots during infection by Lasiodiplodia theobromae was examined. The expression of genes related to metabolic enzymes was also investigated. Control wounded and noninoculated tissue, lesion tissue, and wounded and inoculated surrounding lesion tissue of peach shoots were analyzed. Soluble sugars, glucose, mannose, arabinose, and xylose significantly increased in inoculated tissues of peach shoots compared with control tissues at different times after inoculation. Accumulation of polysaccharides was also observed by section observation and periodic acid Schiff's reagent staining during infection. Analysis using quantitative reverse-transcription polymerase chain reaction revealed that the abundance of key transcripts on the synthesis pathway of uridine diphosphate (UDP)-D-glucuronate, UDP-D-galactose, and UDP-D-arabinose increased but the synthesis of L-galactose and guanosine diphosphate-L-galactose were inhibited. After inoculation, the transcript levels of sugar transportrelated genes (namely, SUT, SOT, GMT, and $U G T$ ) was induced. These changes in sugar content and gene expression were directly associated with peach gum polysaccharide formation and may be responsible for the symptoms of peach gummosis.
Peach gummosis is one of the most important and damaging diseases of peach (Prunus persica L. Batsch) in China, Japan, and the United States $(16,28)$. Peach tree fungal gummosis was first observed in Georgia in 1970 (41). The three species of the genus Botryosphaeria (Lasiodiplodia theobromae, Botryosphaeria dothidea, and Diplodia seriata) were the predominant causes of the disease $(6,39)$. All three species infect through pruning wounds and lenticels $(6,27,42)$. Populations of $L$. theobromae increase in summer (7). L. theobromae discharges ascospores and conidia from diseased peach bark and dead prunings during or soon after rainfall (27). The common symptoms of fungal gummosis are sunken necrotic lesions, which are characteristically swollen and crusty in appearance, and gum exudates from cracked warts on the trunk and major branches (4). Gum exudates are secreted from diseased peach trunks, branches, or fruit; in more serious cases, fungal gummosis results in reduced vigor, small yellow leaves, earlier defoliation, twig die back, and decreased production (16).

The characteristics of peach gum exudate polysaccharide have been studied. Gum polysaccharides have complex components, including many monosaccharides and glycosidic linkages (33). Peach gum polysaccharides are composed of arabinose (Ara), xylose (Xyl), mannose (Man), galactose (Gal), and uronic acids at different molar ratios based on a comparison analysis of peach and nectarine (P. persica var. nucipersica Schneid.) $(34,36)$. The relationship between plant polysaccharides and disease has been

Corresponding author: G. H. Li; E-mail address: liguohuai@mail.hzau.edu.cn

* The $\boldsymbol{e}$-Xtra logo stands for "electronic extra" and indicates that the online version contain three supplemental figures and one supplemental figure. Figures 1 and 5 appear in color online.

http://dx.doi.org/10.1094/PHYTO-01-13-0025-R

(C) 2014 The American Phytopathological Society recently studied. The polysaccharide called Ulva, isolated from green algae, induces defense responses against powdery mildew in cucumber, common bean, and grapevine. Ulva also enhances resistance to powdery mildew in wheat and barley plants by triggering oxidative burst $(13,26)$. In addition, Sun et al. (37) analyzed the polysaccharide composition of intervessel pit membranes and showed that the resistance of grapevine to Pierce's disease depended on the lack of some kinds of polysaccharide. Therefore, polysaccharide formation is closely associated with carbohydrate metabolism and transport.

Carbohydrate components such as soluble sugars, starch, and sucrose display significant variation between inoculated plant tissues and healthy control tissues $(21,27,30)$. These studies suggest that, after pathogen infection, glycometabolism changes to a great extent in plant tissues associated with plant disease. However, the monosaccharide metabolism and the formation mechanisms of peach gum polysaccharides in infected tissues have not been extensively studied. The sucrose transporter (SUT) and sorbitol transporter $(S O T)$ are localized in the phloem of plants and can be responsible for phloem loading $(40,43)$. The transporters of guanosine diphosphate (GDP)-Man and uridine diphosphate (UDP)-Gal are located in the Golgi apparatus, where polysaccharides are formed. These transporters play a critical role in providing substrates for glycosyltransferases and in the synthesis of glycans $(10,22)$.

In this study, current-year peach shoots were inoculated with L. theobromae and the content of several soluble carbohydrates was determined from lesion, tissue surrounding lesions, and healthy tissue during the development of peach gummosis. The relative expression of genes related to sugar metabolism was analyzed. This study aimed to gain a better understanding of peach gummosis and provide a basis to control this disease. Hence, the major challenge was to unravel the changes in carbohydrate metabolism and peach gum polysaccharide formation triggered by L. theobromae in peach shoots. 


\section{MATERIALS AND METHODS}

Plant and pathogen material. Peach plants $(P$. persica L. 'Springsnow') were grown in an experimental field at Huazhong Agricultural University in Wuhan. For all inoculation experiments, current-year shoots were collected from 3-year-old plants of P. persica Springsnow from June to August 2011 and 2012. The plants were grafted on wild peach rootstock. L. theobromae strain JMB-122 was originally isolated from a diseased peach tree in Hubei province, China and maintained in an incubator with a photoperiod of $12 \mathrm{~h}$ of light and $12 \mathrm{~h}$ of darkness at $26^{\circ} \mathrm{C}$ (39).

Inoculation with $L$. theobromae and sample collection. A detached inoculation assay was conducted according to the methods of Britton and Hendrix (6). Strain JMB-122 was cultured on potato dextrose agar (PDA) medium for 3 days prior to inoculation. Current-year shoots were surface sterilized with $70 \%$ alcohol for $10 \mathrm{~s}$, rinsed three times with sterile water, and cut into 15-cm-long segments. The shoot segments were wounded with a sterilized needle, and an actively growing isolate of strain JMB122 on a mycelial agar plug ( $4 \mathrm{~mm}$ in diameter) was placed over the wound. The current-year peach shoots were wounded and inoculated with sterile PDA as controls under the same conditions. All treated shoots were placed in glass bottles containing $100 \mathrm{ml}$ of sterile water, covered with a clear plastic film to maintain humidity, and placed in an incubator. The water in the bottles was changed daily. Unless indicated otherwise, the standard growth conditions were a photoperiod of $12 \mathrm{~h}$ of light $(20,000 \mathrm{lux})$ and $12 \mathrm{~h}$ of darkness at $26^{\circ} \mathrm{C}$ and relative humidity of $90 \%$.

Samples were collected as described by Dorey et al. (8). All samples were collected at the same time point. Control wounded and noninoculated tissues, lesion tissues, and wounded and inoculated surrounding lesion tissues were collected at 0 and $12 \mathrm{~h}$ and 1,2, 3, 4, 5, and 6 days postinoculation (dpi). Control and inoculated tissues were collected within a $0.5-$ to $1.0-\mathrm{cm}$ distance surrounding the wound and lesion, frozen in liquid nitrogen, and stored at $-80^{\circ} \mathrm{C}$ for subsequent use. At each time point, three independent replicates were collected from infected shoots and control shoots, and each replicate consisted of 15 current-year peach shoots for the analysis of carbohydrates and quantitative real-time polymerase chain reaction (qRT-PCR).

Determination of carbohydrates. Sucrose, glucose, fructose, sorbitol, Man, Ara, and Xyl were quantified using a gas chromatography (GC) system (Agilent 6890N, Santa Clara, CA), equipped with an auto-sampler (Agilent 7683), flame ionization detector (FID), HP-5 5\% phenyl-methyl polysiloxane column (30 m by $0.25 \mu \mathrm{m}$ i.d.), and a data processor (Applied Biosystems, Santa Clara, CA). The flow rates of $\mathrm{N}_{2}, \mathrm{H}_{2}$, and air to the FID were 25, 30 , and $40 \mathrm{ml} / \mathrm{min}$, respectively. The GC conditions were set according to Bartolozzi et al. (3), with slight modifications. The injector and detector temperatures were 270 and $300^{\circ} \mathrm{C}$, respectively. The following temperature program was set: from 130 to $152^{\circ} \mathrm{C}$ at $8^{\circ} \mathrm{C} / \mathrm{min}, 152$ to $176^{\circ} \mathrm{C}$ at $12^{\circ} \mathrm{C} / \mathrm{min}, 176$ to $198^{\circ} \mathrm{C}$ at $16^{\circ} \mathrm{C} / \mathrm{min}, 198$ to $238^{\circ} \mathrm{C}$ at $20^{\circ} \mathrm{C} / \mathrm{min}, 239$ to $300^{\circ} \mathrm{C}$ at $24^{\circ} \mathrm{C} / \mathrm{min}$, and finally $300^{\circ} \mathrm{C}$ for $4 \mathrm{~min}$. Each compound was quantified using the internal standard method (3).

For the soluble sugar assay, the content was determined following the anthrone method of Spiro (36).

Periodic acid Schiff's reagent staining. To identify polysaccharides, the samples were cut into 5-by-5-mm pieces. The tangential sections of the peach shoots were cut from control and inoculated tissues (within and near the lesion) at different positions, respectively. Periodic acid Schiff's reagent was used to visualize the polysaccharides of the sectioned materials (1). Sections were incubated in $1 \%(\mathrm{wt} / \mathrm{vol})$ periodic acid $\left(\mathrm{HIO}_{4}\right)$ for $30 \mathrm{~min}$, rinsed in running water for $5 \mathrm{~min}$, then immersed in periodic acid Schiff's reagent for $30 \mathrm{~min}$ in the dark. After staining, the slides were washed three times for 3 min with $2 \%$ (wt/vol) sodium metabisulfite solution and in running water for 5 min. The slides were dried, observed, and photographed using a microscope.

RNA isolation and qRT-PCR. Total RNA from different samples was extracted from $0.5 \mathrm{~g}$ of frozen tissue of peach current-year shoots using an EASYspin Plus RNA kit (Aidlab, Beijing). DNase I (TaKaRa, Dalian, China) was added to remove genomic DNA. RNA yield and purity were analyzed with NANODROP 2000 (Thermo, Waltham, USA), whereas RNA integrity was detected by electrophoresis in $1.5 \%$ agarose gel. First-strand cDNA was synthesized with total RNA using oligo (dT) primers following the manufacturer's instructions on a PrimeScript RT reagent kit (TaKaRa).

Relative expression was determined by performing qRT-PCR in a LightCyler 480 Sequence Detection System (Roche, Basel, Switzerland) using the intercalation dye SYBR Green as a fluorescent reporter. Reactions were carried out in a final volume of $15 \mu$ l containing $30 \mathrm{ng}$ of cDNA, $10 \mu \mathrm{mol}$ of each primer, and $7.5 \mu$ of $2 \times$ SYBR Green PCR master mixes (TaKaRa) according to the manufacturer's instructions. PCR primers were designed based on peach cDNA sequences published in the GenBank database and described in Supplemental Table 1. The thermal cycling conditions were $10 \mathrm{~min}$ of denaturation at $95^{\circ} \mathrm{C}$ followed by 40 cycles of amplification $\left(95^{\circ} \mathrm{C}\right.$ for $15 \mathrm{~s}, 60^{\circ} \mathrm{C}$ for $15 \mathrm{~s}$, and $72^{\circ} \mathrm{C}$ for $\left.1 \mathrm{~min}\right)$. Relative expression levels were analyzed with LightCyler 480 Sequence Detection System software and normalized with the results of actin as a reference. The qRT-PCR results are shown as the mean values of three independent biological replicates \pm standard deviation.

Statistical analysis. The one-way analysis of variance (ANOVA) of SAS program package (version 8.1; SAS Institute, Cary, NC) was used for statistical analysis between inoculated peach shoots and control shoots. Statistically significant differences are reported in the text and shown in the figures.

Accession numbers. Sequence data for the genes in this study can be found in the EMBL/GenBank data libraries under the following accession numbers: L-arabinose kinase (ARA) (KC345021), fructokinase $(F R K) \quad(\mathrm{KC} 339524), \quad$ D-galactokinase $(A G K)$ (KC345020), GDP-Man transporter (GMT) (KC339528), PGI (KC345022), phosphomannomutase (PMM) (KC339527), SUT (KC345023), UDP-D-glucose dehydrogenase (UGD) (KC339532), UDP-glucose 4-epimerase (UGE) (KC339533), UGP (KC339525), UDP-Gal transporter (UGT) (KC339529), UDP-D-Xyl 4-epimerase $(U X E)$ (KC339531), and UDP-D-Xyl synthase (UXS) (KC339530).

\section{RESULTS}

Symptoms. Gum exudates from diseased trunk and branches are a typical symptom of peach gummosis (Fig. 1A and B). The most serious symptom observed was that the trunk and most branches were covered by brown gum exudates (Fig. 1C). The colony morphology of $L$. theobromae strain JMB-122 was cultured on PDA medium for 3 days (Fig. 1D). Under laboratory conditions, a similar symptom was observed in current-year shoot phenotypes at 3 dpi with field symptoms (Fig. 1F), and $129.8 \mathrm{mg}$ of gum exudates was obtained, on average, for every lesion (Supplemental Figure 1). No symptom was observed in control shoots (Fig. 1E). Interestingly, numerous gum exudates were observed from the tangential section surrounding the inoculation point of peach current-year shoots (Fig. 1H), and no symptom was observed in the control shoots (Fig. 1G).

Determination of carbohydrate during the disease. Soluble sugar sucrose, glucose, fructose, sorbitol, Man, Xyl, and Ara production was measured during the infection process of $L$. theobromae in peach current-year shoots and healthy shoots (Figs. 2 and 3). In comparison with the control tissue, the production of sucrose, glucose, and fructose from the lesion decreased after 
inoculation with L. theobromae (Fig. 2A). In tissue surrounding lesion, the soluble sugar level significantly increased at 4 and 5 dpi, except in the gum exudates (Fig. 2A). In addition to the increase in soluble sugar content, glucose significantly increased at 1 and $2 \mathrm{dpi}$, reaching the highest value at 1 dpi compared with the control shoots (Fig. 2C). The sucrose and fructose contents of the tissue surrounding the lesion were significantly lower than those of the controls, although the sucrose content increased at 2 dpi (Fig. 2B and D).

The other sugar distribution patterns (sorbitol, Man, Xyl, and Ara) differed between the controls and inoculated samples throughout the process (Fig. 3A to D, respectively). The sorbitol content of the inoculated surrounding tissues was significantly lower during infection (Fig. 3A). The Man content was higher in the inoculated samples, with significant differences at all inoculation time points (Fig. 3B). Differences in Xyl content between inoculated and control shoots were concentrated at 1, 3, and 4 dpi, with significant increments at 3 and 4 dpi compared with the control shoots (Fig. 3C). The Ara levels were higher in inoculated shoots than uninoculated shoots, with significant differences at 4 and 5 dpi (Fig. 3D).

qRT-PCR of metabolic genes involved in sugar metabolism during the infection process of peach shoots. A simplified metabolic scheme was established, and the expression of genes involved in sugar metabolism in peach shoots was analyzed by qRT-PCR (Fig. 4).

Sucrose, glucose, fructose, and sorbitol metabolism. Neutral invertase (NI) and acid invertase (AI) are involved in sucrose degradation. The relative expression of putative NI and AI was significantly higher in inoculated peach shoots than control shoots at different times after inoculation but their expression patterns were opposite (i.e., NI increased and AI decreased gradually). Regarding the enzymes involved in sucrose synthesis, the expression of sucrose synthase $(S S)$ was significantly higher than that in the control shoots. Sucrose phosphate synthase $(P S S)$ expression only significantly increased at $1 \mathrm{dpi}$, and then no significant decrease was observed in inoculated shoots compared with the control shoots (Supplementa1 Figure 2A).

The transcript levels of NAD-dependent sorbitol dehydrogenase $(S D H)$ significantly increased throughout the course of infection, which was responsible for the degradation of sorbitol. Nevertheless, the expression abundance of sorbitol-6-phosphate dehydrogenase $(S 6 P D H)$ was inhibited during the infection process compared with the controls. Together with the contents of sucrose and sorbitol, they may be used as fuel during infection.

Man and Gal metabolism. D-Man can be synthesized as a free monosaccharide through the fructose pathway (31). Three genes (FRK, phosphoglucose isomerase $[P G I$, and $P M M$ ) involved in the metabolism of Man were assayed in the present research. A significant increase in the expression of $F R K$ was detected at 1 dpi compared with the control shoots. However, the expression level of PGI appeared to be higher at 5 dpi. The transcript levels of $P M M$ also significantly increased at 1 and 5 dpi in inoculated shoots compared with the control shoots. Therefore, the changes in transcript levels had similar patterns with the changes in Man contents.

D-Man serves as the substrate for sugar interconversion enzymes producing GDP-L-Gal or L-Gal. The expression abundance of genes (such as GDP-D-mannose pyrophosphorylase $[G M P]$, GDP-D-Man 3,5-epimerase [GME], L-galactose-1phosphate phosphatase $(G P P)$, and GDP-L-Gal guanylyltransferase $[G G G T]$ ) involved in the L-Gal pathway were inhibited during the infection of peach shoots (Fig. 4). However, compared with the control shoots, the transcript abundance of $U G E$ and $A G K$ involved in UGP-D-Gal significantly increased in inoculated shoots.

Ara and Xyl metabolism. UDP-D-glucuronate is a major branchpoint in the biosynthesis of UDP sugars, including UDP-D-Gal,
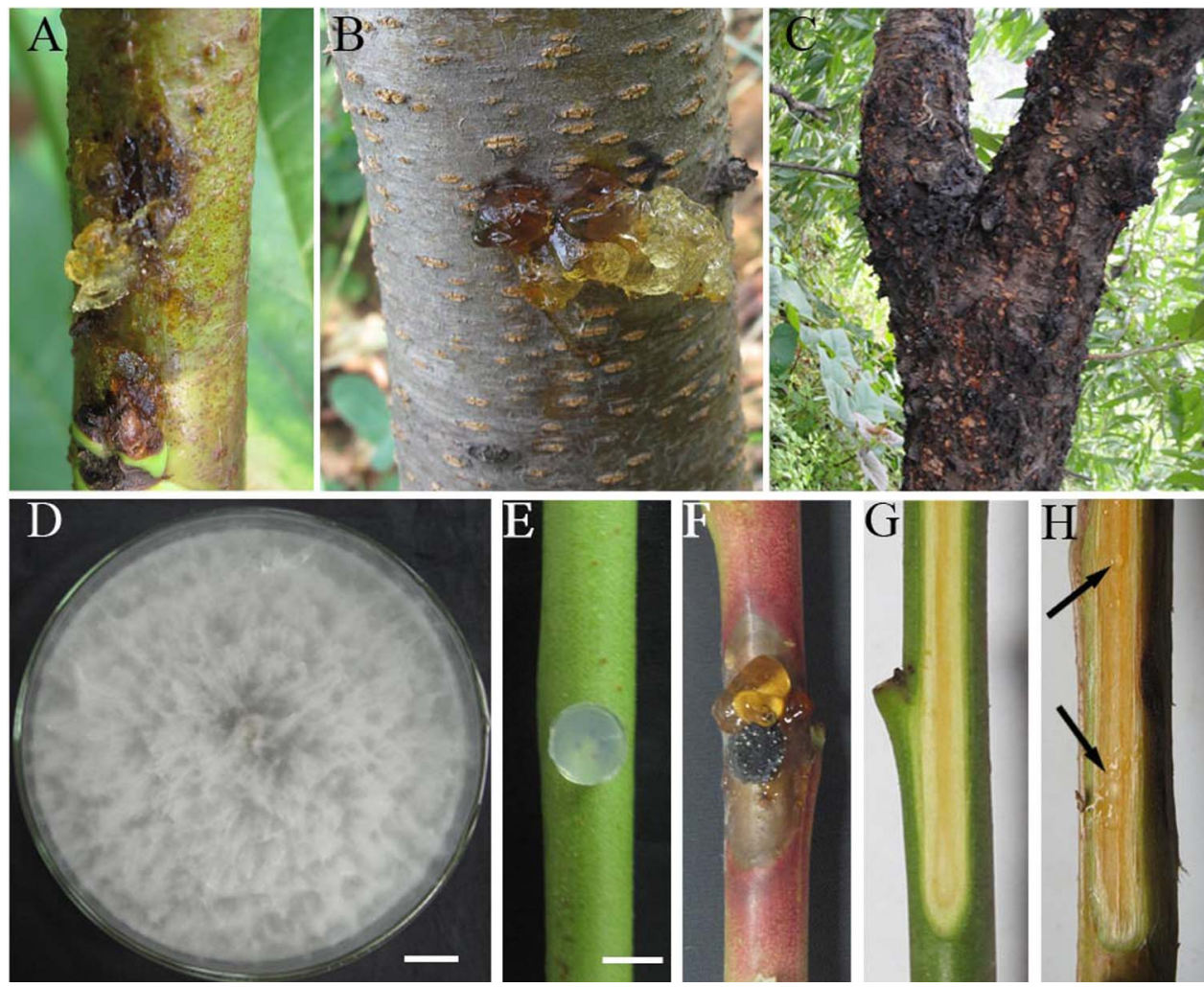

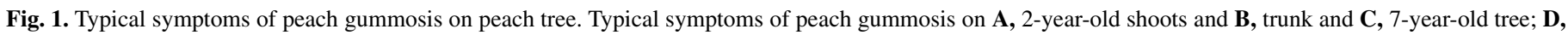

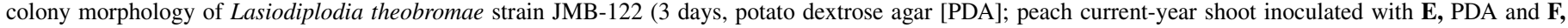

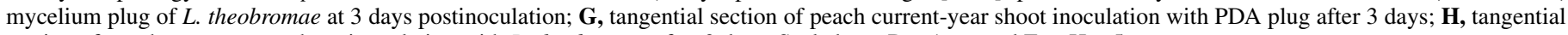
section of peach current-year shoot inoculation with L. theobromae after 3 days. Scale bars: $\mathrm{D}=1 \mathrm{~cm}$ and $\mathrm{E}$ to $\mathrm{H}=5 \mathrm{~mm}$. 

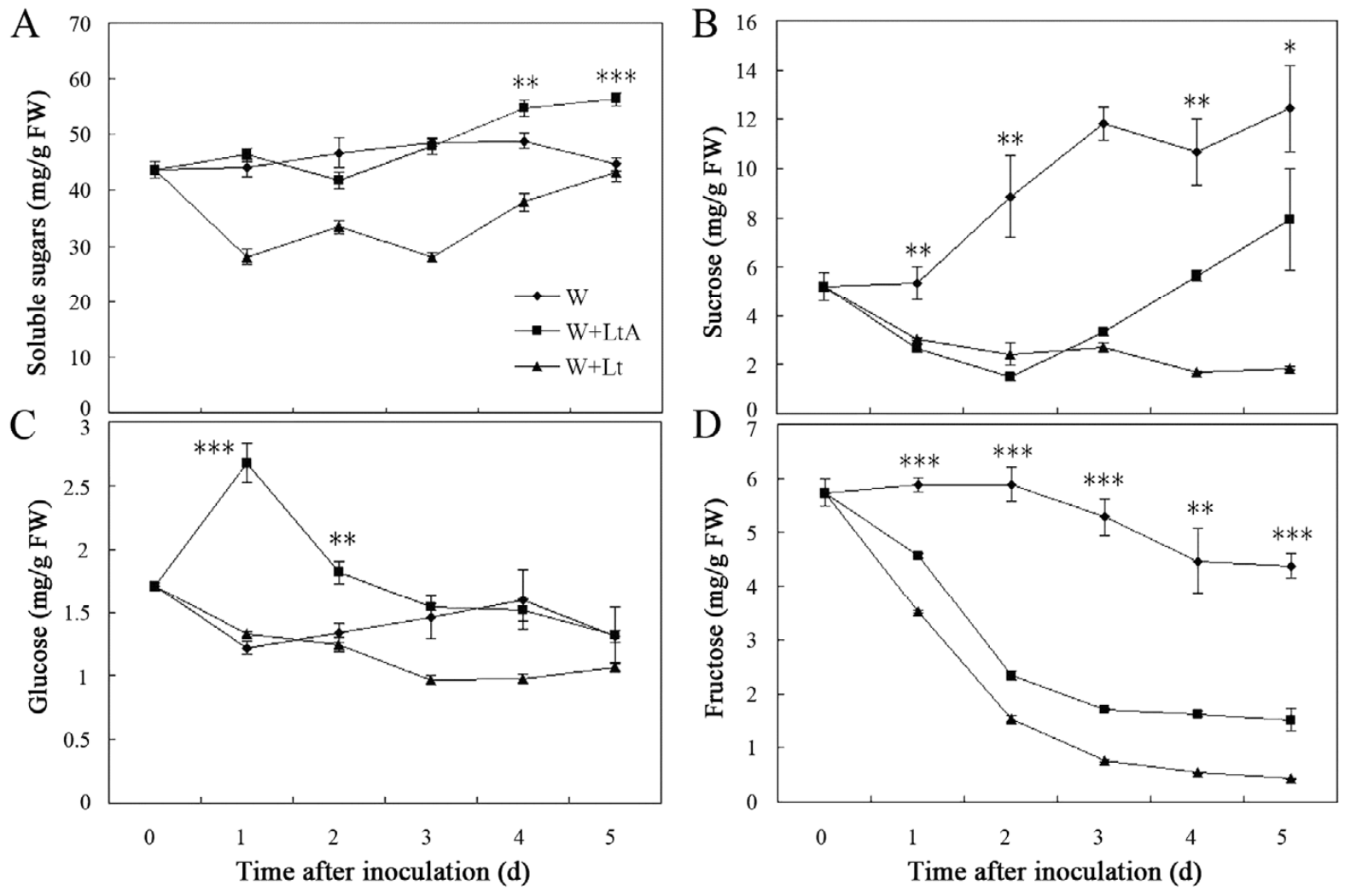

Fig. 2. Production of A, total soluble sugars; B, sucrose; C, glucose; and D, fructose (milligram per gram fresh weight [FW]) in peach shoots from healthy control tissue $(\mathrm{W})$, lesion tissue $(\mathrm{W}+\mathrm{Lt})$, and lesion surrounding tissue $(\mathrm{W}+\mathrm{LtA})$. Values represent the mean of three independent determinations \pm standard deviation at different times after inoculation. Asterisks indicate a significant difference $(*, * *$, and $* * *$ indicate $P<0.05,0.01$, and 0.001 , respectively).
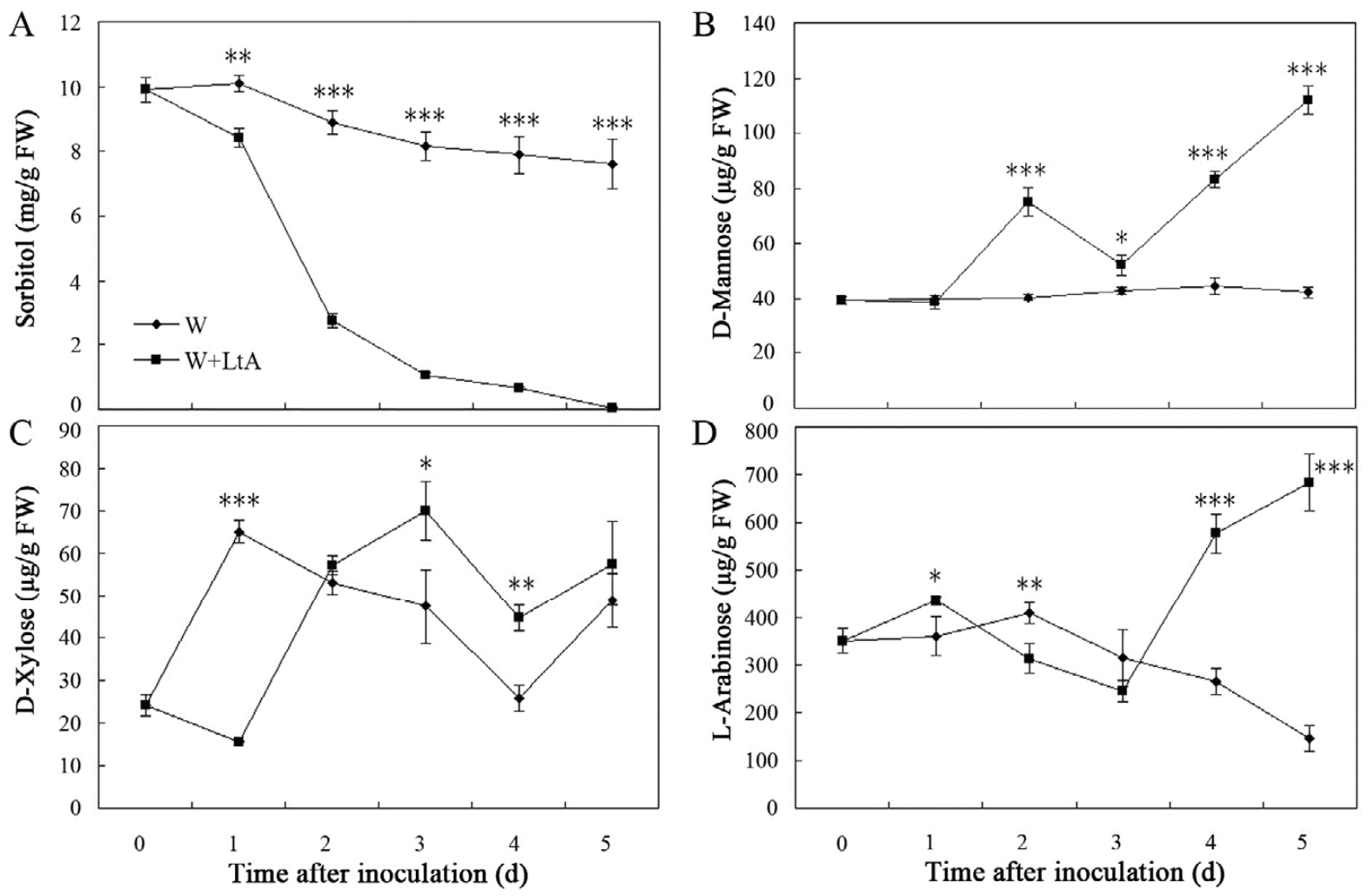

Fig. 3. Levels of A, total sorbitol (milligram per gram fresh weight [FW]); B, D-mannose; C, D-xylose; and D, L-arabinose (microgram per gram FW) in peach shoots from healthy control tissue (W) and lesion surrounding tissue (W+LtA) at different times after inoculation. Values represent the mean of three independent determinations \pm standard deviation at different times after inoculation. Asterisks indicate a significant difference $(*, * *$, and $* * *$ indicate $P<0.05,0.01$, and 0.001 , respectively). 

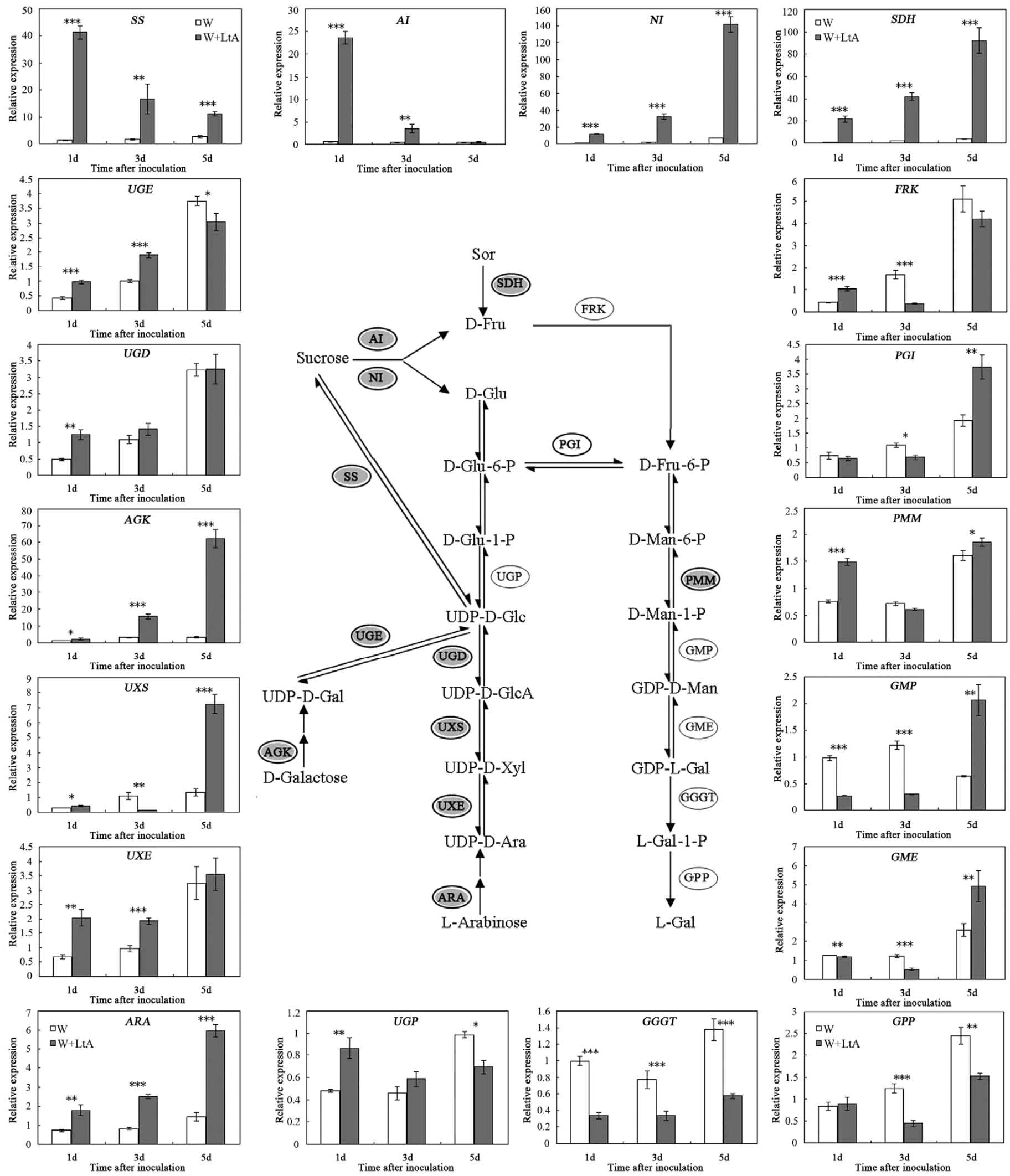

Fig. 4. Simplified metabolic scheme and expression analysis of transcripts of genes involved in sugar metabolism in peach shoots analyzed by quantitative realtime polymerase chain reaction from control tissue (W) and tissue surrounding lesion (W+LtA) an inoculated with Lasiodiplodia theobromae at 1, 3, and 5 days postinoculation (dpi). The relative expression of transcripts of metabolism genes that are increased during infection are indicated in bold with a gray background, whereas the transcripts of metabolism genes that decreased are indicated in normal font with a white background compared with control tissue. The mRNA levels of the genes were normalized to the transcript of actin in the same sample (fold change $1=$ time $0 \mathrm{dpi}$ ). Means of the results obtained using three independent RNAs as templates are shown. Standard deviations are shown. Asterisks indicate a significant difference $(*, * *$, and $* * *$ indicate $P<0.05,0.01$, and 0.001 , respectively). 
UDP-Ara, and UDP-Xyl (29). In this biosynthesis pathway, the transcript level of $U G D$ increased by $\approx 2.6$-fold in inoculated shoots at 1 dpi compared with the control shoots. The relative expression of $U X S$ increased with significant differences throughout the infection course.

UDP-L-Ara is synthesized from UDP-D-Xyl by reversible $U X E$. Accordingly, the relative expression of $U X E$ increased to a greater extent in inoculated shoots than in the control shoots at 1 and 3 dpi. On the other hand, the expression level of $A R A$ increased throughout the infection course, with 2.5-, 3.3-, and 4.1-fold changes at 1,3 , and $5 \mathrm{dpi}$, respectively, compared with the control shoots.

Accumulation and transport of sugars. The current-year shoots infected with $L$. theobromae began to produce gum at $3 \mathrm{dpi}$ under laboratory conditions. Interestingly, excluding the gum exudates, a significant increase in the soluble sugar level was observed at 4 and 5 dpi in inoculated shoots compared with uninoculated shoots (Fig. 2A). We observed that the tangential section of inoculated shoots contained more gum from the parenchyma cells of phloem than the control shoots (Fig. 1D and E). In addition, the parenchyma cells of the phloem showed more prunosus in inoculated shoots than the control shoots through periodic acid Schiff (PAS) staining. This finding indicated that more polysaccharides accumulated in the parenchyma cell of peach phloem in inoculated shoots (Fig. 5). These data suggested that the pronounced increase in soluble sugars and parts of monosaccharide levels were associated with polysaccharide formation during the infection of $L$. theobromae in the peach current-year shoots.

Four genes involved in sugar transport were analyzed in peach shoots during the disease process by qRT-PCR (Fig. 6). The levels of SUT increased by nearly 1.4 - and 1.8 -fold at 1 and 5 dpi, respectively, whereas no significant difference was observed at 3 dpi (Fig. 6A). Almost 1.5- and 2.6-fold increases in the transcript level of $S O T$ were found at 3 and 5 dpi, respectively, compared with the control shoots (Fig. 6B). The transcript level of GMT significantly increased 3.3-, 1.3-, and 1.7-fold at 1, 3, and $5 \mathrm{dpi}$, respectively, in inoculated peach shoots compared with the controls (Fig. 6C). The transcript levels of $U G T$ only significantly increased 1.5-fold at 3 dpi (Fig. 6D).

\section{DISCUSSION}

Peach gummosis caused by Botryosphaeria spp. infection results in very distinctive gum exudates in peach trunks and shoots. In this work, we confirmed that inoculated detached peach shoots had the same symptoms as peach shoots under field conditions (Fig. 1). After infection with Botryosphaeria spp., necrosis and death of infected tissues were triggered by unknown factors and processes (39). Previous reports have suggested that programmed cell death is induced as a restriction for pathogen infection (17). The surrounding cells of the hypersensitive response lesion were stimulated at the same time, leading to metabolic changes, decreased mRNA levels, and defensive signals. Different zones far from the lesion displayed different spatial and temporal expression of defense responses (8). Thus, the peach shoot lesion and the tissue surrounding the lesion were separately collected to evaluate the sugar distribution patterns.

Partial changes in sugar metabolites were analyzed during the infection process of L. theobromae (Fig. 2). Although the sucrose, glucose, and fructose levels in lesions and surrounding tissues decreased, the amounts of soluble sugars increased after 3 dpi. These changes can be associated with the disintegration of dead cells and the degradation of cell wall polysaccharides (32). Nevertheless, sorbitol, Man, Ara, and Xyl in lesions were not detected, possibly because their amounts were very low in the samples (Fig. 3).

In the tissues surrounding the lesion, the concentrations of soluble sugars, Man, Xyl, and Ara significantly increased from 3 to $5 \mathrm{dpi}$, whereas the glucose amounts only exhibited a notable increase at 1 and 2 dpi. The high content of soluble sugars, glucose, and sucrose in cocoa have been observed after infection with Crinipellis perniciosa (30). Our results support previous ones that the soluble sugar content of inoculated tissues significantly increases in comparison with noninoculatted tissues. Gum exudates were first observed in the current-year shoots at $3 \mathrm{dpi}$ (Fig. 1). The Man and Ara contents were at a lower level in inoculated tissues at 3 dpi compared with the control tissues. By contrast, the concentrations of soluble sugars and sucrose showed a lower level at $2 \mathrm{dpi}$. This finding can be attributed to the fact that sucrose cleavage is vital to multicellular plants for the

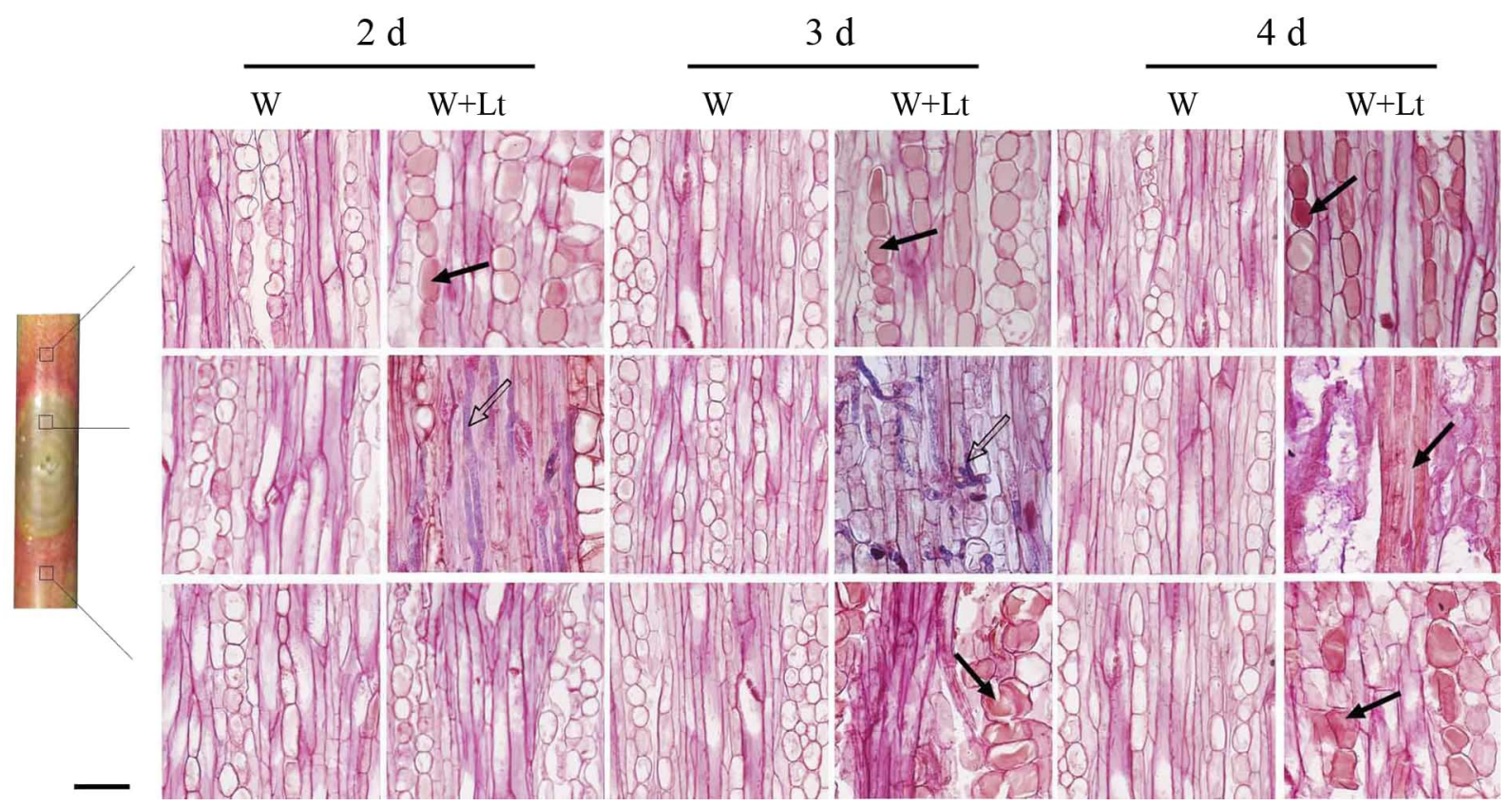

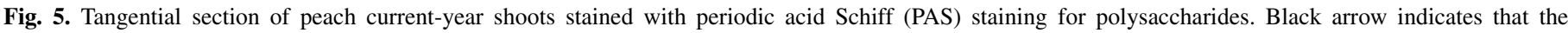

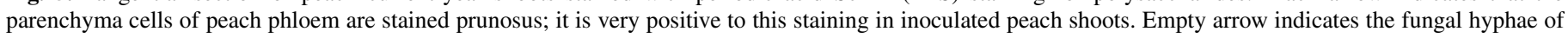
Lasiodiplodia theobromae. $\mathrm{W}=$ control wounded and noninfected tissue and $\mathrm{W}+\mathrm{Lt}=$ wounded and infected tissue. Scale bars $=30 \mu \mathrm{m}$. 
allocation of crucial carbon resources and for the initiation of hexose-based sugar signals (15). The pathways for the synthesis of Xyl and Ara are catalyzed by sucrose synthase, which converts UDP and sucrose into UDP-D-glucose and fructose, whereas the pathway for the synthesis of Man involves the conversion pathway of fructose (29). The relative expression levels of $S S$ and $U G P$ showed a similar result and significantly increased after inoculation (Fig. 4). In summary, the increased amount of several monosaccharides (Ara, Man, and Xyl) in inoculated peach shoots was consistent with the composition of gum exudate polysaccharides of peach trees.

Our results showed that the synthesis of L-Gal and GDP-L-Gal was inhibited after inoculation with $L$. theobromae. However, the relative expression levels of critical genes involved in the synthesis of UDP-D-Gal notably increased (Fig. 4). This phenomenon can be explained by the fact that UDP-D-Gal was ultimately needed in the Golgi apparatus for the synthesis of cell wall polysaccharides and chloroplast galactolipids (14). However, GDP-L-Gal has been confirmed to contribute to the pathway to L-ascorbate biosynthesis (12). Alternatively, GDP-L-Gal may not be uniformly distributed throughout the Golgi stacks with other monosaccharides (29). Thus, the high molecular weight polysaccharides of the cell wall played a role in the outcome of hostpathogen interactions (38). Therefore, UDP-D-Gal was more important for peach gum polysaccharides than GDP-L-Gal.

The expression of four genes involved in sugar transport was analyzed, and results confirmed that sugar transport was enhanced after shoots were inoculated with L. theobromae (Fig. 6). Moreover, the accumulation of polysaccharides in inoculated tissues as observed by section observation and PAS staining supported this conclusion (Figs. 1E and 5). These data suggested that the changes in and pathways of glycometabolism were regulated in peach shoots during infection with $L$. theobromae (Supplemental Figure 3). The accumulation of monosaccharides may be due to the change in and transformation of the glycometabolism pathway. Polysaccharides were synthesized by certain types of mono- saccharides in the Golgi apparatus and transported by the endomembrane system.

Sugars can contribute to immune responses and function as priming molecules (20). A phenomenon termed "high-sugar resistance" involves high levels of sugars for enhancing defense against pathogens and decreasing susceptibility (11). Previous reports have shown that pretreatment of rice plants with sucrose enhances resistance to Magnaporthe oryzae infection (9). Some polysaccharides also induce a defense response in tomato plants for wilt and enhanced resistance in wheat against powdery mildew $(19,23)$. Therefore, biodegradable sugar-like extracts may be used to counteract peach gummosis from high-sugar-content plants (such as sugarcane and beet) and some polysaccharides (such as peach gum), which may be cheaper and safer than chemical pesticides. Most importantly, peach trees avoid waste of sugars against pathogen infection.

In summary, various polysaccharide elicitors were characterized from pathogens and host plants (25). Some polysaccharides can act as signal molecules to elicit defensive responses and protect plants against phyotopathogenic fungi $(2,24)$. Furthermore, sugars such as sucrose, glucose, and fructose are involved in many metabolic and signal pathways that interact with hormonal pathways. They also contribute to immune responses against pathogens $(5,20)$. Gum exudates mainly comprise polysaccharides containing different monosaccharides at different molar ratios $(33,35)$. Plant gum exudates appeared to act as protective agents in response to microbial invasion (18). However, this phenomenon cannot be sufficient to explain why, in peach gummosis, a large amount of gum exudates is continually produced by peach trees without a regulation mechanism. The changes in carbohydrate content and expression of genes related to carbohydrates may indicate the formation of peach gum polysaccharide in response to L. theobromae infection.

The relationship between peach gum polysaccharide and glycometabolism is important. Therefore, to confirm polysaccharide formation and gain further insight into peach-L. theobromae
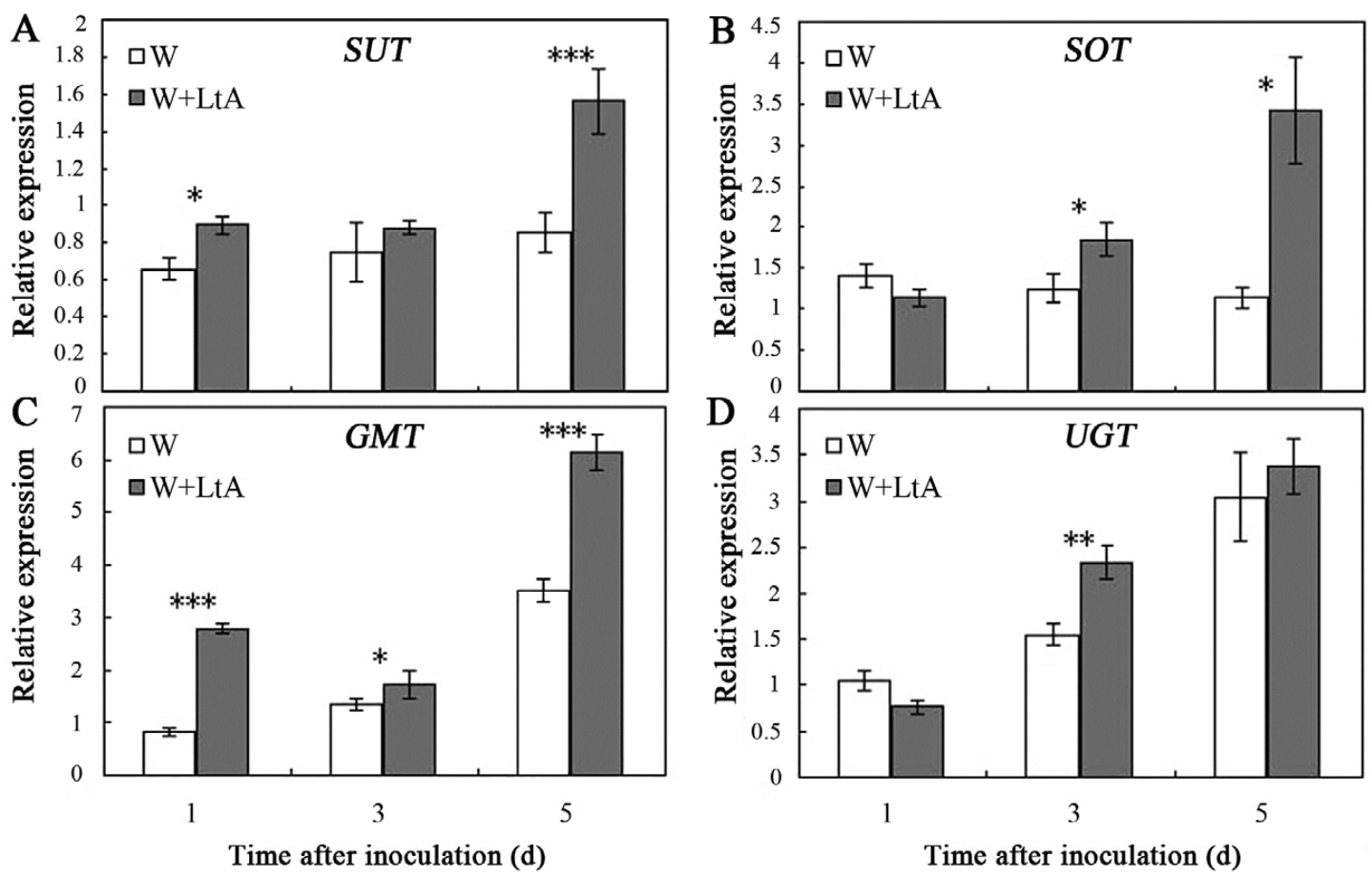

Fig. 6. Relative expression analysis of transcripts of sugars transporter in peach shoots infected with Lasiodiplodia theobromae and healthy control shoots with quantitative real-time polymerase chain reaction. A, SUT = sucrose transporter; $\mathbf{B}$, SOT $=$ Sorbitol transporter; $\mathbf{C}$, GMT = GDP-mannose transporter; and $\mathbf{D}$, UGT = UDP-galactose transporter. mRNA levels of the genes were normalized to the transcript of actin in the same sample (fold change $1=$ time 0 days postinoculation). Relative values are the means of three biological replicates \pm standard deviation. Asterisks indicate a significant difference $(*, * *$, and $* * *$ indicate $P<0.05,0.01$, and 0.001 , respectively). 
interaction, additional biochemical studies on enzyme activity involved in glycometabolism and detailed analysis on histopathology are required. The pathogenicity factors and fungal metabolism of $L$. theobromae are also critical in understanding polysaccharide formation during this process.

\section{ACKNOWLEDGMENTS}

This research was supported by the National Natural Science Foundation of China (grant number 31272120) and China Agriculture Research System (grant number CARS-31). We thank J.-T. Xie for revising the manuscript.

\section{LITERATURE CITED}

1. Aarrouf, J., Garcin, A., Lizzi, Y., and EI-Maataoui, M. 2008. Immunolocalization and histocytopathological effects of Xanthomonas arboricola pv. pruni on naturally infected leaf and fruit tissues of peach (Prunus persica L. Batsch). J. Phytopathol. 156:338-345.

2. Aziz, A., Poinssot, B., Daire, X., Adrian, M., Bezier, A., Lambert, B., Joubert, J. M., and Pugin, A. 2003. Laminarin elicits defense responses in grapevine and induces protection against Botrytis cinerea and Plasmopara viticola. Mol. Plant-Microbe Interact. 16:1118-1128.

3. Bartolozzi, F., Bertazza, G., Bassi, D., and Cristoferi, G. 1997. Simultaneous determination of soluble sugars and organic acids as their trimethylsilyl derivatives in apricot fruits by gas-liquid chromatography. J. Chromatogr. A 758:99-107.

4. Biggs, A. R., and Britton, K. O. 1988. Presymptom histopathology of peach trees inoculated with Botryosphaeria obtuse and B. dothidea. Phytopathology 78:1109-1118.

5. Bolouri-Moghaddam, M. R., Le Roy, K., Xiang, L., Rolland, F., and Van den Ende, W. 2010. Sugar signalling and antioxidant network connections in plant cells. FEBS J. 277:2022-2037.

6. Britton, K. O., and Hendrix, F. F. 1982. Three species of Botryosphaeria cause peach tree gummosis in Georgia. Plant Dis. 66:1120-1121.

7. Britton, K. O., and Hendrix, F. F. 1986. Population dynamics of Botryosphaeria spp. in peach gummosis cankers. Plant Dis. 70:134-136.

8. Dorey, S., Baillieul, F., Pierrel, M. A., Saindrenan, P., Fritig, B., and Kauffmann, S. 1997. Spatial and temporal induction of cell death, defense genes, and accumulation of salicylic acid in tobacco leaves reacting hypersensitively to a fungal glycoprotein elicitor. Mol. Plant-Microbe Interact. 10:646-655.

9. Gomez-Ariza, J., Campo, S., Rufat, M., Estopa, M., Messeguer, J., Segundo, B. S., and Coca, M. 2007. Sucrose-mediated priming of plant defense responses and broad-spectrum disease resistance by overexpression of the maize pathogenesis-related PRms protein in rice plants. Mol. Plant-Microbe Interact. 20:832-842.

10. Handford, M. G., Sicilia, F., Brandizzi, F., Chung, J. H., and Dupree, P. 2004. Arabidopsis thaliana expresses multiple Golgi-localised nucleotidesugar transporters related to GONST1. Mol. Gen. Genomics 272:397-410.

11. Horsfall, J. G., and Dimond, A. E. 1957. Interactions of tissue sugar, growth substances and disease susceptibility. Z. Pflanzenkrankh. Pflanzenschutz 64:415-421.

12. Imai, T., Ban, Y., Terakami, S., Yamamoto, T., and Moriguchi, T. 2009. L-Ascorbate biosynthesis in peach: cloning of six L-galactose pathwayrelated genes and their expression during peach fruit development. Physiol. Plant. 136:139-149.

13. Jaulneau, V., Lafitte, C., Corio-Costet, M.-F., Stadnik, M. J., Salamagne, S., Briand, X., Esquerre-Tugaye, M.-T., and Dumas, B. 2011. An Ulva armoricana extract protects plants against three powdery mildew pathogens. Eur. J. Plant Pathol. 131:393-401.

14. Joyard, J., Teyssier, E., Miege, C., Berny-Seigneurin, D., Marechal, E., Block, M. A., Dorne, A. J., Rolland, N., Ajlani, G., and Douce, R. 1998. The biochemical machinery of plastid envelope membranes. Plant Physiol. 118:715-723.

15. Koch, K. 2004. Sucrose metabolism: Regulatory mechanisms and pivotal roles in sugar sensing and plant development. Curr. Opin. Plant Biol. 7:235-246.

16. Li, H. Y., Cao, R. B., and Mu, Y. T. 1995. In vitro inhibition of Botryosphaeria dothidea and Lasiodiplodia theobromae, and chemical control of gummosis disease of Japanese apricot and peach trees in Zhejiang Province, China. Crop Prot. 14:187-191.

17. Liu, Y. L., Schiff, M., Czymmek, K., Talloczy, Z., Levine, B., and DineshKumar, S. P. 2005. Autophagy regulates programmed cell death during the plant innate immune response. Cell 121:567-577.

18. Marques, M. R., and Xavier-Filho, J. 1991. Enzymatic and inhibitory activities of cashew tree gum exudate. Phytochemistry 30:1431-1433.

19. Milling, A., Babujee, L., and Allen, C. 2011. Ralstonia solanacearum extracellular polysaccharide is a specific elicitor of defense responses in wilt-resistant tomato plants. PLoS One 6:e15853.

20. Moghaddam, M. R. B., and Ende, W. V. 2012. Sugars and plant innate immunity. J. Exp. Bot. 63:3989-3998.

21. Ndoumou, D. O., Ndzomo, G. T., and Djocgoue, P. F. 1996. Changes in carbohydrate, amino acid and phenol contents in cocoa pods from three clones after infection with Phytophthora megakarya Bra and Grif. Ann. Bot. Lond. 77:153-158.

22. Norambuena, L., Nilo, R., Handford, M., Reyes, F., Marchant, L., Meisel, L., and Orellana, A. 2005. AtUTr2 is an Arabidopsis thaliana nucleotide sugar transporter located in the Golgi apparatus capable of transporting UDP-galactose. Planta 222:521-529.

23. Omokolo, N. D., Nankeu, D. J., Niemenak, N., and Djocgoue, P. F. 2002. Analysis of amino acids and carbohydrates in the cortex of nine clones of Theobroma cacao L. in relation to their susceptibility to Phytophthora megakarya Bra. and Grif. Crop Prot. 21:395-402.

24. Ortmann, I., Conrath, U., and Moerschbacher, B. M. 2006. Exopolysaccharides of Pantoea agglomerans have different priming and eliciting activities in suspension-cultured cells of monocots and dicots. FEBS Lett. 580:4491-4494.

25. Ortmann, I., and Moerschbacher, B. M. 2006. Spent growth medium of Pantoea agglomerans primes wheat suspension cells for augmented accumulation of hydrogen peroxide enhanced peroxidase activity upon elicitation. Planta 224:963-970.

26. Paulert, R., Ebbinghaus, D., Urlass, C., and Moerschbacher, B. M. 2010. Priming of the oxidative burst in rice and wheat cell cultures by ulvan, a polysaccharide from green macroalgae, and enhanced resistance against powdery mildew in wheat and barley plants. Plant Pathol. 59:634-642.

27. Pusey, P. L. 1989. Availability and dispersal of ascospores and conidia of Botryosphaeria in peach orchards. Phytopathology 79:635-639.

28. Reilly, C. C., and Okie, W. R. 1982. Distribution in the southeastern United States of peach tree fungal gummosis by Botryosphaeria dothidea. Plant Dis. 66:158-161.

29. Reiter, W. D., and Vanzin, G. F. 2001. Molecular genetics of nucleotide sugar interconversion pathways in plants. Plant Mol. Biol. 47:95-113.

30. Scarpari, L. M., Meinhardt, L. W., Mazzafera, P., Pomella, A. W. V., Schiavinato, M. A., Cascardo, J. C. M., and Pereira, G. A. G. 2005. Biochemical changes during the development of witches' broom: The most important disease of cocoa in Brazil caused by Crinipellis perniciosa. J. Exp. Bot. 56:865-877.

31. Seifert, G. J. 2004. Nucleotide sugar interconversions and cell wall biosynthesis: how to bring the inside to the outside. Curr. Opin. Plant Biol. 7:277-284

32. Shin, M. H., Lee, D. Y., Skogerson, K., Wohlgemuth, G., Choi, I.-G., Fiehn, O., and Kim, K. H. 2010. Global metabolic profiling of plant cell wall polysaccharide degradation by Saccharophagus degradans. Biotechnol. Bioeng. 105:477-488.

33. Simas, F. F., Barrzaz, R. R., Petkowicz, C. L. O., Silveira, J. L. M., Sassaki, G. L., Santos, E. M. R., Gorin, P. A. J., and Iacomini, M. 2010. Rheological and structural characteristics of peach tree gum exudate. Food Hydrocolloid. 24:486-493.

34. Simas, F. F., Gorin, P. A. J., Wagner, R., Sassaki, G. L., Bonkerner, A., and Iacomini, M. 2008. Comparison of structure of gum exudate polysaccharides from the trunk and fruit of the peach tree (Prunus persica). Carbohydr. Polym. 71:218-228.

35. Simas, F. F., Wagner, R., Santos, E. M. R., Sassaki, G. L., Gorin, P. A. J., and Iacomini, M. 2009. Polysaccharide of nectarine gum exudate: Comparison with that of peach gum. Carbohydr. Polym. 76:485-487.

36. Spiro, R. G. 1966. Analysis of sugars found in glycoproteins. Pages 3-26 in: Methods in Enzymology, Vol. VIII, Complex Carbohydrates. E. S. Neufeld and V. Ginsburg, eds. Academic Press, New York.

37. Sun, Q., Greve, L. C., and Labavitch, J. M. 2011. Polysaccharide compositions of intervessel pit membranes contribute to Pierce's disease resistance of grapevines. Plant Physiol. 155:1976-1987.

38. Vorwerk, S., Somerville, S., and Somerville, C. 2004. The role of plant cell wall polysaccharide composition in disease resistance. Trends Plant Sci. 9:203-209.

39. Wang, F., Zhao, L., Li, G., Huang, J., and Hsiang, T. 2011. Identification and characterization of Botryosphaeria spp. causing gummosis of peach trees in Hubei province, central China. Plant Dis. 95:1378-1384.

40. Watari, J., Kobae, Y., Yamaki, S., Yamada, K., Toyofuku, K., Tabuchi, T., and Shiratake, K. 2004. Identification of sorbitol transporters expressed in the phloem of apple source leaves. Plant Cell Physiol. 45:1032-1041.

41. Weaver, D. J. 1974. A gummosis disease of peach trees caused by Botryosphaeria dothidea. Phytopathology 64:1429-1432.

42. Weaver, D. J. 1979. Role of conidia of Botryosphaeria dothidea in the natural spread of peach tree gummosis. Phytopathology 69:330-334.

43. Williams, L. E., Lemoine, R., and Sauer, N. 2000. Sugar transporters in higher plants-A diversity of roles and complex regulation. Trends Plant Sci. 5:283-290. 